\title{
Senghor, Kourouma et Sony Labou Tansi : D'un mouvement littéraire à la littérature en mouvement ou la Négritude de l'ère postcoloniale
}

\author{
N. André Siamundele \\ Colby College
}

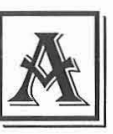

nalyser l'évolution de la création littéraire en Afrique francophone constitue non seulement un regard critique vis-à-vis des thèmes développés par des écrivains durant près d'un siècle mais aussi un exercice qui prend en compte le rapport entre le créateur et la langue d'expression. En effet, si nous prenons simplement en guise d'exemple les propos tenus par nos trois écrivains par rapport à la langue française. Nous aurons la grande admiration de Senghor :

Le français, ce sont les grandes orgues qui se prêtent à tous les timbres, à tous les effets, des douceurs les plus sauvages aux fulgurances de l'orage. Il est tout à tout et en même temps, flûte, hautbois, trompette, tambours ${ }^{1}$.

Alors que pour Sony Labou Tansi :

J'écris en français parce que c'est dans cette langue-là que le peuple dont je témoigne a été violé. Je me souviens de ma virginité. Et mes rapports avec la langue française sont des rapports de force majeure oui, finalement. Il faut dire que s'il y a du français et de moi quelqu'un qui soit en position de force, ce n'est pas le français, c'est moi. Je n'ai pas eu recours au français c'est lui qui a recours à moi $^{2}$.

1 Senghor, L.S., " Négritude et Humanisme » Liberté III, Paris : Seuil, 1964: 167.

2 Labou Tansi, S., "Un citoyen de ce siècle " Equateur, n 1, 1986: 30.

Siamundele 9 
Entre ces deux positions, Ahmadou Kourouma a réussi à écrire l'Afrique en français. Il a su injecter dans la langue française des réalités intraduisibles car pour lui " la littérature, ce n'est pas la langue 3 . » Ce qui fait d'ailleurs que son héros dans Allah n'est pas obligé, utilise le français à la manière malinké.

Ainsi lorsque nous parlons de passer le flambeau, il s'agit bien évidemment de se situer par rapport à un parcours historique qui permet le passage d'une étape à une autre sans établir une rupture tranchée mais en préservant le sens de la continuité. Il s'agit en fait de la rupture dans la continuité. Ce mouvement de renouvellement d'énergie intellectuelle et créatrice répond à un besoin de changement de rythme, fruit d'un contexte socio-historique particulier.

En tant que mouvement littéraire, la Négritude définit une manière de s'exprimer suivant un contexte historico-social bien indiqué. Elle suggère à la fois une prise de conscience, une remise en question, une révolution, une rupture et une identité alors que la littérature en mouvement est une force qui gouverne la littéraire face aux frustrations et aux situations de malaise. Il s'agit d'une vive réaction face à une réalité complètement délabrée et déphasée. Il est question d'une réalité qui n'a pas su livrer les attentes annoncées. C'est la situation chaotique que vit la postcolonie, c'est-à-dire comme l'écrit Achille Mbembe, "period that is far from being uniform and absolutely cannot be reduced to a succession of moments and events, but in which instants, moments, and events are, as it were, on top of one another, inside one another ${ }^{4}$. " Ainsi, face à cette situation de désordre, c'est une écriture déchaînée qui se met en mouvement. L'écriture senghorienne n'était pas déchaînée, elle s'installait ou simplement prenait place dans l'histoire alors que pour Kourouma et Labou Tansi, la littérature doit pouvoir se libérer totalement.

Par rapport à la négritude première, celle d'aujourd'hui possède une agressivité provoquée par de longues années de domination. La négritude est donc changeante, elle possède une dimension historique que Senghor n'explicite pas, mais dont il est conscient. Avec la Négritude, il s'agit de s'affirmer et d'affirmer l'identité nègre.

3 Ahmadou Kourouma, Interview à RFI, le 5 septembre 2001.

4 Mbembe, Achille, On the Postcolony, London : University of California Press, $2001: 242$. 


\section{Senghor}

Dans Ce que je crois, Senghor définit la Négritude comme « l'ensemble des valeurs de la civilisation noire5. "

Il importe de saisir la poésie de Senghor comme un moment important de libération. Elle tient à libérer des contraintes et à définir et à établir une identité nègre. L'imagination poétique aura donc permis à la Négritude d'envisager une réalité hors de la réalité de l'époque contre laquelle le mouvement s'opposait.

Il définit déjà sa négritude avec des mots forts et très engageant :

Ma Négritude point n'est sommeil de la race Mais soleil de l'âme, ma négritude vue et vie Ma Négritude est truelle à la main, est lance Au poing. Recade. Il n'est question de boire, de Manger l'instant qui passe.

[...]

Ma tâche est d'éveiller mon peuple aux futurs flamboyants

Ma joie de créer des images pour le nourrir, ô lumières Rythmées de la Parole!

(Élégie des Alizés) $^{6}$

L'on saisit en filigrane chez Senghor, l'esprit de révolte et d'affirmation qui prendra corps dans l'écriture en Afrique francophone. Il s'affirme et s'engage.

Il promet de ne point « boire, de manger l'instant qui passe ». Il ne s'agit pas d'être simple témoin de l'histoire, il faut saisir les armes à sa disposition pour se lancer dans ce combat d'affirmation de soi. Il prend sa " lance au poing » car sa tâche, reconnaît-il, est « d'éveiller son peuple aux futurs flamboyants ». Au fond, Senghor s'inscrit dans une lignée qui sera celle de faire porter à la parole et à l'image le poids de l'identité. Il réussit admirablement lorsqu'il injecte dans ses poèmes des forces pour soutenir les idées d'indépendances et de bonheur.

5 Senghor, L.S. Ce que je crois : Négritude, Francité et Civilisation de l'Universel, Paris : Grasset, $1988: 153$.

6 Senghor, L.S. Elégies majeures suivi de Dialogue sur la Poésie francophone, Paris : Seuil, $1979: 13$. 
C'est sur ce caractère fonctionnel de sa poésie que s'appuie Senghor, car au bout du tunnel, il perçoit une libération qui va au-delà de la simple contemplation de la langue française. C'est par la séduction et l'admiration que Senghor est entré dans cette langue mais il a fini par mettre cette dernière à son service. Il a servi la langue en lui apportant un ton poétique particulier. La poésie de Senghor s'inscrit dans un contexte social qui reste et demeure fortement marqué par des injustices, le silence et l'exploitation dont est victime l'homme noir. Elle constitue l'expression artistique la plus engageante et certainement la plus profondément engagée car elle séduit et emporte. Les poèmes senghoriens tout en exaltant les valeurs culturelles africaines en français marquent un pas vers le dépassement de la confrontation entre la civilisation occidentale et la culture africaine. Ainsi, fait-il parler la France et l'Afrique :

Jusqu'en Sine jusqu'en Seine, est dans mes veines fragiles, mon sang irréductible ("In Memoriam ", Chants d’Ombre)

Il y a une volonté d'appropriation lorsque le poète par un travail d'association mémorielle fait revivre l'Afrique à travers un regard parisien. Sine revient à l'existence culturelle par le contact visuel que le poète établit avec la Seine.

De même, l'on trouvera dans un autre poème, l'écho des souvenirs d'Afrique en face de la présence européenne :

Tout le long du jour, durement secoué sur les bancs du train de ferraille et poussif et poussiéreux

Me voici cherchant l'oubli de l'Europe au cour pastoral

Du Sine

("Tout Le Long Du Jour " in Chants d'Ombre)

Et le célèbre vers du poème " Joal " :

Joal

Je me rappelle.

[...]

Quelle marche lasse le long des jours d'Europe où parfois Apparaît un jazz orphelin qui sanglote sanglote sanglote (" Joal " in Chants d'Ombre) 
Présenté comme le symbole de la réussite de la mission civilisatrice en Afrique, Senghor a été également été au centre et même en avant-plan d'un mouvement littéraire et culturel qui a désavoué la colonisation à travers une poésie qui exaltait les valeurs négro-africaines. Tout ceci malgré son attachement profond aux valeurs de la grande civilisation française.

Essentiellement, nous retrouvons dans le fond de la créativité senghorienne, un réel besoin d'intégrer les souvenirs heureux de l'Afrique dans la composition poétique en français. L'écriture et la pensée senghoriennes sont caractérisées par les éléments qui demeurent présents aujourd'hui dans la littérature africaine francophone. Mais il est vrai aussi qu'il y a un dépassement par rapport aux thèmes de la négritude. L'affirmation du Noir n'est plus au premier plan car le glissement vers l'affirmation de soi dans un contexte de post-indépendance s'impose.

\section{Kourouma}

De Senghor à Kourouma : on passe de la poésie au roman. En effet, l'écriture de Kourouma demeure fortement marquée par une poéticité qui prend corps dans le terroir même de son Malinké natal. Autant Senghor pour dire sa Négritude avait besoin de la langue de l'Autre, Kourouma force la langue de l'Autre à rendre des réalités qui étaient jusque-là étrangères à elle. Ces deux écrivains sont tous les deux francophiles — j'entends par là leur contribution et non leur amour aveugle vis-à-vis du Français — à des niveaux différents. Ainsi pour réussir dans sa mission, Kourouma a eu besoin d'apporter des innovations scripturales dans son premier roman Les soleils des Indépendances. Il est parvenu à faire plier la langue coloniale devant les exigences de la source d'inspiration de son ceuvre. "Je tire mon inspiration de l'Afrique, des problèmes africains, reconnaitt-il7 "

En s'appuyant sur l'Afrique pour écrire ses textes, Kourouma tient à souligner les spécificités qui fondent ce continent. Il insiste : « Il faut sauver les mythes africains en les employant dans les romans. Chaque fois qu'un mythe disparaît, c'est toute l'humanité qui perd quelque chose. "

La rupture vis-à-vis du français s'organise déjà avec Les soleils des Indépendances qui commence ainsi : "Il y avait une semaine qu'avait fini dans la capitale Kone Ibrahima, de race malinke, ou disons-le en malinke : il n'avait pas soutenu un petit rhume... ${ }^{8}$ Le lecteur se trouve en face d'un

7 Interview à Radio France Internationale.

8 Kourouma, A, Les soleils des indépendances, Paris : Seuil : 9. 
texte en français mais réellement aspergée de la saveur malinké. Et du coup, l’on voit Kourouma s'éloigner de la pensée et de l'écriture senghoriennes. Cet éloignement constitue aussi une distance car le flambeau se transmet sur fond d'un contexte socio-historique caractérisé par les indépendances politiques des pays africains. Senghor cède le flambeau de la Négritude au moment où la libération est établie et consacrée avec les indépendances tandis que Kourouma le saisit pour dire le malaise qui a suivi ladite libération. C'est véritablement avec Kourouma que l'on voit prendre forme le paradoxe : libération/malaise que cachent mal les indépendances.

Kourouma saisit le fondement de la Négritude et ajoute une autre dimension à la course vers le bonheur et la libération. Il s'éloigne de la langue française alors même qu'il choisit de s'enraciner dans les valeurs culturelles africaines. Il s'éloigne de cette langue qui a permis de cadrer son message, son combat et sa philosophie. C'est dire qu'entre Kourouma et Senghor le rapport à la langue française ne se vit pas de la même manière.

Dans un autre roman Allah n'est pas obligé, il y a la prise en compte du rapport qui s'établit à la fois entre le narrateur et la langue française mais aussi entre le lecteur et le discours littéraire. En plaçant au centre de l'intrigue la situation combien actuelle et fortement triste des enfants soldats dans les armées africaines, le texte de Kourouma pose la question de la nécessité et de l'incompatibilité des corps linguistiques dans le contexte post-colonial. Au-delà de son contenu, c'est réellement un discours symbolique de rejet vis-à-vis de la langue française qui se lit sous la plume d'Ahmadou Kourouma : "Suis p'tit nègre. Pas parce que suis black et gosse. Non! Mais suis p'tit nègre parce que je parle mal le français?. "

C'est par la langue, instrument (outil) de communication que Birahima choisit de s'identifier. Et il se définit par une négation partielle et nuancée. Il n'est point par ce qu’il est, il est par ce qu’ul n'est pas. Ce degré d'appropriation de "parle mal le français » lui donne une licence de s'exprimer librement sans se buter à la rigidité des règles de la langue. Dans cette démarche, il parvient à établir des zones floues car Kourouma arrive à nous présenter deux textes : l'un est écrit et l'autre est dit. Tel est l'univers dans lequel Birahima taille son chemin pour l'affirmation de son identité. Tout au long du texte, ce personnage central ne se définit point par ses caractéristiques biologiques mais par son rapport à une langue mal apprivoisée.

9 Kourouma, A, Allah n'est pas obligé, Paris : Seuil, 2000 : 9. 
C'est donc par la langue que Birahima entre dans le monde extérieur. Il n'a pas peur de la détruire, il la met à son service et non le contraire. Il a un esprit méthodique particulier :

- la langue : il la parle mal

- l'école : il l'a quittée car elle ne sert à rien.

Il dit notamment :

"L'école ne vaut pas le pet de la grand-mère parce que, même avec la licence de l'université, on n'est pas fichu d'être infirmier ou instituteur dans une des républiques bananières corrompues de l'Afrique francophone. (République bananière signifie apparemment démocratique, en fait régie par des intérêts privés, la corruption) » 10

L'on saisit de la part de cet enfant-soldat une prise de conscience vis-àvis du désordre instauré et institutionnalisé par les adultes. De ce fait, l'enfant-soldat cesse de s'inscrire dans le schéma conventionnel de ce que représente l'enfance en même temps qu'il consacre la rupture linguistique située à la frontière entre l'enfance et l'âge adulte.

Ce texte a la particularité de reconnaître la présence d'un corps étranger d'une nature étrangère. Tout le récit consiste en une lutte permanente entre l'acceptation de ce corps et l'affirmation d'une identité. Ce qui soulève bien entendu la question du rejet avec les conséquences nuisibles et dévastatrices que cela implique. À partir du moment où l'enfant prend les armes, cela entraîne la remise en question manifeste de l'idée même de l'enfant. Un enfant soldat est un soldat qui a cessé d'être un enfant. Le terme " enfant » se définit ainsi par sa négation et par une procédure qui le vide totalement de sa substance. L'enfant-narrateur soumet la langue française aux réalités capricieuses de l'Afrique des dictateurs et des guerres civiles.

Considérant le texte littéraire comme un corps, la rupture linguistique s'inscrit dans un cadre global de coexistence entre deux entités en lutte permanente : le corps et la greffe. Ainsi, Kourouma n'a pas seulement marqué la rupture au niveau littéraire en 1967, il a bouleversé une certaine idée de la langue et du discours pour toujours. La greffe discursive constitue une expérience de métissage, de rencontre qui instaure la mise sur pied d'un ordre nouveau sur le plan de l'identité. Un aspect important de

10 Kourouma, A, Allah n'est pas obligé, Paris : Seuil, $2000: 10$. 
cette procédure greffale, c'est la recherche de l'harmonie entre deux termes qui ferait surgir un troisième dans lequel les deux premiers se reconnâitraient sans qu'il n'y ait de rejet. Or le discours déjà prend une certaine distance vis-à-vis de la langue.

Encore une fois, Ahmadou Kourouma vient de marquer l'Histoire en établissant une cassure, une rupture, un dépassement des frontières établies. Il vient de montrer que l'Afrique peut et devrait en fait se concevoir en dehors des divisions fallacieuses et précaires telles que francophonie et anglophonie. Autant, il tourne en dérision le Libéria des Américains, il se moque également des nègres africains indigènes francophones qui disent merde! putain! salaud! au lieu d'employer des mots malinke comme Faforo, Gnamokodé. Emprunter des interjections étrangères pour exprimer des sentiments comme la colère, le mépris ou l'impatience indique clairement que l'idée de soi se noie tragiquement dans l'identité de l'autre.

Dans ce texte de Kourouma, la langue française - à travers mots et expressions - fonctionne comme une greffe qui contribue à la survie du récit de Birahima. Non seulement Birahima affirme qu'il n'est qu'un enfant à cause de son âge, il affirme aussi qu'il ne l'est pas à cause de ses expériences : "j'ai tué beaucoup de gens avec kalachnikov et me suis bien camé avec kanif et les autres drogues dures ${ }^{11}$. "En effet, sans les greffes imposés aux textes, on ne saurait véritablement saisir les nuances, le message et la portée de ce qui constitue l'élément central du récit à savoir la question des enfants-soldats.

\section{Sony Labou Tansi}

Alors que l'Ivoirien Ahmadou Kourouma fait passer un discours malinké en français, l'écrivain congolais Sony Labou Tansi met la langue française à l'épreuve en la fissurant. Son premier roman La Vie et demie ne se laisse pas dompter à la première lecture, il constitue une créativité narrative et un défi à plus d'un titre. Les noms des protagonistes s'imbriquent dans des dénominations des lieux au point qu'il devient difficile de percevoir une ligne droite et claire dans le déroulement du récit. On comprend dès lors que la création littéraire de Sony Labou Tansi constitue l'émergence de plusieurs rencontres et niveaux de signification. Ce roman peint avec une violence rare la cruauté démesurée du régime dictatorial du guide providentiel Matela-Pene Loango.

11 Kourouma, A, Allah n'est pas obligé, Pairs : Seuil, 2000 : 11.

\section{LittéRéalité}


On se croirait facilement en face d'une écriture qui abuse du verbe, mais au fond ce sont les innombrables maux du monde qui épuisent la substance du verbe, ainsi que nous le rappelle Sony :

J'écris, ou je crie, un peu pour forcer le monde à venir au monde [...] J'estime que le monde dit moderne est un scandale et une honte, je ne dis que cette chose-là en plusieurs maux. ${ }^{12}$

À la sortie de La Vie et demie en 1979, les critiques ont reconnu certes une rupture sous forme d'innovation par rapport à une écriture africaine traditionnellement linéaire et limpide. L'un d'eux écrit notamment :

Cet écrivain est manifestement en marge de la chose littéraire, préférant bâtir son œuvre loin de tout cet appareil un peu terrifiant et à la longue stérilisant où les discussions n'en finissent pas et où l'on sacre et sabre à qui mieux ${ }^{13}$.

Mais ont-ils réellement senti et saisi jusqu'où pouvait conduire cette révolution? Ainsi à propos de Sony Labou Tansi, Célestin Monga écrira :

le non-conformisme de son écriture libérée et impudique, qui ne s'embarrasse jamais de guillemets pour exprimer le quotidien [...] Les thèmes de Labou Tansi - les contradictions de l'Afrique moderne - ne sont pas nouveaux. Mais ils font l'objet d'un traitement particulier qui évite l'ennui au lecteur ${ }^{14}$.

L'auteur de La Vie et demie a non seulement bouleversé radicalement le contenu du roman africain, mais il lui a aussi imposé une forme à la mesure de la violence de son message. Il faut dès lors accepter que "les problèmes posés dans son ouvre ont été, certes, déjà représentés par d'autres romanciers, mais la nouveauté réside dans le traitement artistique que subit la réalité dans son écriture ${ }^{15}$. "Il s'agit d'une écriture de la

12 Labou Tansi, S, L'Etat honteux, Paris : Seul, $1981: 5$.

13 Edouard Maunick, "Sony Labou Tansi : l'homme qui dit tous les hommes " in Demain l'Afrique, $\mathrm{n}^{\circ} 40$, (19 décembre 1979) : 81.

14 Célestin Monga, "Sony Labou Tansi ou l'écriture non conformiste " in Jeune Afrique, no 1217, (2 mai 1984) : 61.

15 Landry-Wilfrid Miampika, "Sony Labou Tansi : l'anti-modernité des États 
dénonciation car le monde de Sony Labou Tansi se dévoile dans l'isomorphisme à la fois entre l'écrit et le cri, et entre les maux et les mots. L'écriture devient ce moule qui donne une forme particulière à une réalité ayant plusieurs facettes. Suivant une vision bien singulière telle que définie par Sony Labou Tansi, la littérature s'arroge un pouvoir de dénonciation loin du simple projet esthétique. Ainsi construit-il son écriture :

Je regarde les rues, les places, le monde autour de moi. Le tout est enveloppé de silence. Cela me donne toujours envie de crever le silence. Spirituellement, cela est horrible de se taire devant l'intolérable. Alors j'écris comme on crie. Il y a trop de silence en notre monde. Crever le silence, c'est vivre. Le silence qu'impose l'argent, le silence de l'histoire, le silence qu'imposent les fusils... Il faut le tuer parce qu'il sera notre autorisation de respirer ${ }^{16}$.

Le combat contre le silence semble être une préoccupation cardinale de la création laboutansienne. On ne peut s'empêcher de percevoir un projet destiné à combler un vide. L'écriture de Sony Labou Tansi insiste autant sur 'ce qu'il faut dénoncer que sur la manière de le faire. C'est dans l'avertissement de son premier roman qu'il donne sans équivoque la mesure et les contours de ses objectifs :

Moi qui vous parle de l'absurdité de l'absurde, moi qui inaugure l'absurdité du désespoir - d'où voulez-vous que je parle sinon du dehors? À une époque où l'homme est plus que jamais résolu à tuer la vie, comment voulez-vous que je parle sinon en chair-motsde-passe ${ }^{17}$ ?

Loin de constituer une exception, la marginalité se présente comme norme chez cet écrivain congolais. Il se définit non pas comme un "auteur engagé " mais bien comme un " homme engageant". Avec lui, l'écriture devient comme dirait Roland Barthes une fonction, c'est-à-dire qu' " elle est le rapport entre la Création et la Société; elle est le langage littéraire

honteux ou la parenthèse de la mocherie " in Colloque Sony Labou Tansi et Sylvain Bemba (Corbeil-Essonnes : Ices, 1996) : 65.

16 Sony Labou Tansi, « Locataires de la même maison » in Diagonales, $\mathrm{n}^{\circ}$ 9, (1989) : 3-4.

17 Labou Tansi, S, La vie et demie, Paris : Seuil, 1979 : 9. 
transformé par sa destination sociale, elle est la forme saisie dans son intention humaine et liée aux grandes crises de l'Histoire ${ }^{18}$. "

Dans l'hommage qu'il lui adresse, Kadima-Nzuji relève que Sony Labou Tansi :

dépliait une écriture faite de ruptures et de retournements, fondée essentiellement sur l'invention verbale, l'emploi de néologismes et de verbes décalés, l'acclimatation de la langue de création à la logique de son univers mental et affectif. Comme chez Kourouma, l'écriture romanesque de Sony consacrait la primauté des anachronies sur la linéarité du récit, de l'émiettement sur l'homogénéité de l'espace, de l'imaginaire sur la description réaliste du vécu africain. Elle laissait ainsi augurer un renouvellement profond du discours romanesque africain ${ }^{19}$.

Il a été démontré plus d'une fois que Labou Tansi a été fort marqué dès son enfance par la tradition Kongo. Comme l'écrit Jean-Michel Dévésa :

L'horizon de Sony ignorait la rhétorique classique des genres et le strict cloisonnement des sphères d'activité. L'écrivain se situait résolument ailleurs : au carrefour de deux mondes, celui que lui ont légué ses pères et celui que l'Histoire lui a imposé20.

Dans son ouvrage intitulé Sony Labou Tansi : Écrivain de la honte et des rives magiques du Kongo, Jean-Michel Devesa démontre que " toute sa vie, Sony Labou Tansi a donc crié (en kikongo lookd) la honte de son pays en français, ce qui impliquait d'envoûter (en kikongo, loka) la langue de Victor Hugo et de s'en emparer pour lui faire exprimer la détresse et l'humiliation de son peuple ${ }^{21}$. » Ses rapports avec la langue française s'inscrivent dans le cadre d'une douce confrontation car il réinvente la langue qu'il considère

18 Roland Barthes, Le Degré zero de l'écriture suivi de Eléments de Sémiologie, (Paris : Seuil, 1953 et 1964) : 17.

19 Mukala Kadima-Nzuji, "Sony Labou Tansi tel que je l'ai connu », Présence Francophone, no 52, 1998, 9-10.

20 Jean-Michel Devesa, "Le Kongo mental de Sony Labou Tansi ", in Colloque Sony Labou Tansi et Sylvain Ntari Bemba: 7.

21 Jean-Michel Devesa, Sony Labou Tansi. Ecrivain de la honte et des rives magiques du Kongo, (Paris : L'Harmattan, 1996) : 324. 
plus comme une référence que comme un instrument de révérence. C'est ainsi qu'il n’hésite pas à affirmer notamment :

J'ai toujours dit que nous nommons les mêmes choses... Quand un Français nomme un fleuve, c'est la Seine, c'est Garonne. Et ici, la Garonne, c'est une rivière... Le Congo, c'est autre chose... Si tous les deux nous disons "fleuve", il y en a un qui ment. Alors, moi, pour ne pas mentir, je suis obligé d'insuffler quelque chose au mot, de le mettre dans un certain déséquilibre... Et c'est voulu ${ }^{22}$.

Dans ses œuvres, le français sert de support à des réalités qui prennent racine dans les vibrations profondes de la culture Kongo. Au cours d'un entretien, il indique la nécessité d'établir une perspective particulière vis-àvis de la langue française :

Il est déjà emmerdant pour un Africain de lire un livre, parce que forcément forme de mort. Il est plus emmerdant de le lire en français et il l'est davantage de l'écrire dans cette langue, à moins de passer le hic en faisant éclater cette langue frigide qu'est le français, c'est-à-dire en essayant de lui prêter la luxuriance et le pétillement de notre tempérament tropical, les respirations haletantes de nos langues et la chaleur folle de notre moi vital, vitré. Le français, je peux me tromper, me paraît être une langue de raison contrairement à la langue de ma mère qui est une langue de respiration (soit dit entre nous que la respiration raisonne pour ne pas tomber dans le panneau séculaire de ceux qui scindent le monde en gens qui raisonnent et gens qui dansent). Bref, la frigidité de la langue française (qui peut être soignée) m’est rendue évidente par la naissance du ou des créoles africains et antillais ${ }^{23}$.

L'écriture africaine francophone est une écriture de contestation. Contestation vis-à-vis d'une langue française qui se veut orthodoxe, normative et même dogmatique. Contestation ironique face à un pouvoir qui abuse du verbe au point de lui enlever toute sa valeur signifiante.

22 Jean-Michel Devesa, "Sony Labou Tansi et le théâtre : la maïeutique d'un enfant du Pool » inPrésence francophone, $\mathrm{n}^{\circ} 52: 24$.

23 Sony Labou Tansi, cité par Georges Ngal, «Les tropicalités de Sony Labou Tansi » in Silex, no 23, (4e trimestre 1982) : 134. 
En effet, il n'est plus possible d'ignorer la responsabilité de l'écrivain face aux vicissitudes de l'histoire. Il n'a pas le luxe de demeurer muet. C'est ainsi qu'il se met au premier plan et devient le porte-étendard d'une conscience qui prend forme dans une société en mal d'identité. Il y a un appel pressant de dénonciation. Il faut procéder au décloisonnement des questions liées à l'identité car " c'est sous la pression de l'histoire et de la tradition que s'établissent les écritures possibles d'un écrivain donné24. »

L'écriture devient une arme privilégiée pour faire passer un message qui risque de ne pas être entendu autrement, ainsi que le souligne Sony Labou Tansi :

Je vous parle tout simplement avec la seule force des mots, au moment où quelque chose de grave peut les mettre en grossesse d'une hantise. Quand les mots ont bu un verre d'angoisse, et qu'ils se mettent subitement à tituber, à tergiverser et à tâtonner sans vergogne $[\ldots]$ Nous devons les uns et les autres apprendre à mettre à l'heure toutes les pendules de l'histoire, de la morale, de la raison, du rêve, de l'intelligence [...] Dans un monde où la justice est périmée, le droit sent mauvais. [...] Nous sommes arrivés à ce moment crucial où nous devons apprendre à tout réinventer : les concepts, les approches, les habitudes, les méthodes, les outils, les nations, les espaces... tout aux jours d'aujourd'hui est à réinventer. [...] On n'est pas un homme libre sans avoir acquis tous les visas de la responsabilité. [...] En parlant de puissance, vous est-il possible de réaliser après tant de vanité et d'arrogance, que vous n’avez puissance qu'à déséquilibrer? [...] Quelle est la place de la pauvreté dans un monde qui a mal au ventre? Quelle est la place de la liberté dans un monde sans responsabilité25?

L'écriture littérature s'impose comme un acte audacieux ainsi que l'affirme ce personnage de La Vie et demie : "s'il le faut qu'il écrive avec son sang s'il veut vraiment écrire » (LVD : 83). L'écriture littéraire devient ainsi un prétexte sous forme de porte d'entrée dans un monde d'une pluralité de signification. Ainsi que l'écrit Roland Barthes : «L'écriture est précisément ce compromis entre une liberté et un souvenir, elle est cette liberté

24 Roland Barthes, Le degré zéro de l'écriture, : 24.

25 Sony Labou Tansi, «Lettre fermée aux gens du nord et compagnie » in L'Evénement européen. Dialogues eurafricains, no 19 (septembre 1992) : 24. 
souvenante qui n'est liberté que dans le geste du choix, mais déjà plus dans sa durée ${ }^{26}$. »

\section{Conclusion}

L'émancipation, l'indépendance, le développement ou la liberté ne sauraient se limiter à des formes artificielles et superficielles de pensée. Il existe dans la profondeur de tout acte d'affirmation de soi un élan de fierté qui lutte constamment avec des forces destructrices d'assimilation et d'assujettissement. Déjà Léopold Sédar Senghor avait vu le malaise du consommateur :

On peut transporter telle quelle chez nous l'organisation politique et sociale de la métropole, avec départements et députés, prolétariat et partis, syndicats et enseignement laïc [...] on risque seulement de faire de nous [...] des consommateurs, non des producteurs de culture. Car la vigne, c'est un exemple entre mille, ne peut s'acclimater en Afrique noire; elle y pousse, mais les raisins n'arrivent pas à maturité. C'est que le sol est autre, et autre le climat ${ }^{27}$.

Les textes sont des foyers qui expriment une forme de désarroi que le réel vit. On en arrive à considérer l'engagement comme un élément primordial de l'écriture dans la mesure où l'écrivain se soumet à un travail de composition basée sur des traces, c'est-à-dire des signes linguistiques qui renvoient à tout un univers référentiel précis. À travers le parcours, il y a un phénomène de normalisation de la langue française qui fait que les mots cessent d'être innocents, ils sont chargés de signification à la fois culturelle et linguistique. Ainsi le support matériel de l'écriture littéraire trahit à la fois une forme déterminée d'engagement et un regard sur soi qui s'interroge sur la problématique essentielle de l'identité. C'est dans ce cadre que de Senghor à Sony Labou Tansi, il est plausible de suivre une littérature, vecteur des idées, qui poursuit historiquement et sûrement son chemin de force de dénonciation et d'affirmation. Il existe certainement un pont, un lien entre ces trois écrivains. L'essentiel est de ne point se laisser prendre par les moments de désaccord qui ne fait que mettre en mouvement la littérature africaine.

26 Roland Barthes, Le degré zero de l'écriture : 24.

27 Jean-Pierre Biondi, Senghor ou la tentation de l'universel: Paris : Denoël : 31. 